\title{
Produtividade de madeira do eucalipto correlacionada com atributos do solo visando ao mapeamento de zonas específicas de manejo
}

\author{
Eucalyptus wood productivity correlated with soil attributes aiming the mapping of management \\ specifics regions
}

\author{
Morel de Passos e Carvalho ${ }^{I}$ Veridiana Zocoler de MendonçaII \\ Francisco Cezar Belchor Lages Pereira ${ }^{\text {II }}$ Marcelo Valentini Arf ${ }^{\text {II }}$ Claudinei Kappes ${ }^{\text {II }}$ \\ Flávio Carlos Dalchiavon"II
}

\section{RESUMO}

A correta intervenção espacial na administração da lavoura, decorrente das zonas específicas de manejo do solo, aumenta a produtividade e a lucratividade agrícola. No ano de 2010, em Selvíria, Mato Grosso do Sul, Brasil $\left(20^{\circ} 20^{\prime}\right.$ $S$ lat.; $51^{\circ} 24^{\prime}$ W long.) foram empregadas correlações, espaciais $e$ de Pearson, entre dados da planta de eucalipto e alguns atributos físico-químicos do solo, visando encontrar aquele que se correlacionasse bem com a produtividade de madeira. Para tanto, instalou-se uma rede geoestatística para a coleta de dados de solo e planta, com 120 pontos amostrais, num talhão de Eucalyptus camaldulensis de 2 anos. O solo foi um Latossolo Vermelho distrófico (Haplic Acrustox) com declive de $0,025 \mathrm{~m} \mathrm{~m}^{-1}$. O diâmetro basal da copa e a densidade do solo, por resultarem estreitas correlações com o volume de madeira, evidenciaram zonas específicas de manejo do solo intimamente associadas à produção de madeira do eucalipto.

Palavras-chave: manejo do solo, agricultura de precisão, Eucalyptus camaldulensis.

\section{ABSTRACT}

The perfect spatial intervention to manage the cultivation of the land, deriving from specific regions of the soil mapping, increases the agricultural productivity, as well as its clear gain. The relationships, spatial and Pearson, between eucalyptus data plant with some physical and chemical attributes of soil, in the growing season of 2011, in Selviria County, Mato Grosso do Sul State, Brazil (20 20' S lat.; $51^{\circ} 24^{\prime}$ W long.), were studied in order to obtain the one that could have the best relationship in order to improve the wood productivity. Thus, a geostatistical grid was installed for the soil and plant data collection, it contained 120 sample points, in plantation of
Eucalyptus camaldulensis of 2 years old. The soil was a Dystrophic Red Latosol (Haplic Acrustox) whit slope of $0.025 \mathrm{~m}$ $m^{-1}$. Due to their excellent relationship with wood productivity, the basis diameter of the crown and the bulk density showed management specific regions of soil whit high relation to eucalyptus wood productivity.

Key words: soil management, precision agriculture, Eucalyptus camaldulensis.

\section{INTRODUÇÃo}

Atualmente, no Brasil, o Eucalyptus spp é a essência florestal que mais atende as necessidades de reposição de matéria-prima para a fabricação de papel. Sua plantação ocupa 3,5 milhões de hectares, produzindo-se em média $45 \mathrm{~m}^{3} \mathrm{ha}^{-1}$ ano $^{-1}$ de madeira. $\mathrm{O}$ Eucalyptus camaldulensis é uma espécie de grande interesse econômico. Possui boa capacidade de crescer em solos pobres, resistência a longos períodos de seca, tolerância ao excesso de chuvas, bem como produz madeira dura e pesada (LIMA et al., 2010).

A agricultura de precisão (AP) determina o "exato" manejo da lavoura tendo como base o mapeamento de zonas específicas de manejo do solo. No Brasil, encontra-se em fase de adoção, na qual o agricultor tenta elaborar mapas de produtividade agrícola que possuam elevada correlação espacial com os mapas dos nutrientes vegetais. Seus maiores

IPrograma de Pós-graduação em Agronomia, Departamento de Fotossanidade, Engenharia Rural e Solos (DEFERS), Faculdade de Engenharia de Ilha Solteira, Universidade Estadual Paulista "Júlio de Mesquita Filho" (FEIS/UNESP), Av. Brasil, 56, Centro, 15385-000, Ilha Solteira, SP, Brasil. E-mail: morel@agr.feis.unesp.br. Autor para correspondência.

IIDEFERS, FEIS/UNESP, Ilha Solteira, SP, Brasil.

IIIInstituto Federal de Mato Grosso (IFMT), Campo Novo do Parecis, MT, Brasil. 
benefícios são a redução do custo de produção devido ao menor gasto com insumos e o aumento da produtividade agrícola (TSCHIEDEL \& FERREIRA, 2002; MOLIN et al., 2007). Assim, tendo como exemplo determinado adubo, seria possível aplicá-lo com taxas variáveis às referidas zonas. Naquelas onde ocorressem os menores teores nutricionais, normalmente associadas às mais baixas produtividades agrícolas, deveriam ser empregadas as mais elevadas taxas, sendo o inverso verdadeiro. Portanto, esse é o aspecto que decisivamente diferencia a agricultura de precisão da convencional, que efetua a aplicação do adubo com base na média global da área cultivada.

A geoestatística analisa a dependência espacial de dados georreferenciados, aos quais é ajustado o semivariograma, representado pelo gráfico da semivariância em função das distâncias entre observações. A partir dele pode-se confeccionar o mapa de krigagem para cada atributo pesquisado, do solo ou da planta, que representa a variabilidade espacial dos dados, tendo isso sido efetuado por ROSA FILHO et al. (2011) para o E. camaldulensis. Contudo, havendo afinidade entre as dependências espaciais de dois atributos quaisquer, modelada pelo semivariograma cruzado, pode-se obter o mapa de cokrigagem para o atributo principal, de difícil obtenção e de maior interesse, em função do atributo secundário, normalmente de muito fácil obtenção (MOLIN et al., 2007; LIMA et al., 2010). Poder-se-ia, então, a partir do atributo secundário, obter as zonas específicas de manejo do solo para o atributo primário.

Assim, o objetivo do presente trabalho, realizado num talhão de eucalipto sob um Latossolo Vermelho distrófico de Selvíria (MS), foi caracterizar as zonas específicas de manejo do solo, empregando correlações espaciais e de Pearson entre produtividade de madeira com alguns atributos físico-químicos do solo, no sentido de se indicar aquele que mais eficientemente esteja relacionado com o aumento da mencionada produtividade.

\section{MATERIAL E MÉTODOS}

O estudo foi desenvolvido em 2010, na Faculdade de Engenharia de Ilha Solteira - UNESP (SP), em Selvíria (MS, Brasil), latitude 20²0’31' S, longitude $51^{\circ} 24^{\prime} 12$ " We altitude de $363 \mathrm{~m}$. O clima é A (tropical úmido com estação chuvosa no verão $\mathrm{e}^{\mathrm{w}}$ seca no inverno). O solo, classificado conforme a EMBRAPA (2006), foi um LATOSSOLO VERMELHO Distrófico típico argiloso, A moderado (Haplic Acrustox), com declive de $0,025 \mathrm{~m} \mathrm{~m}^{-1}$. Na camada $0-0,40 \mathrm{~m}$ apresentava, em 10/07/2010: 1) macroporosidade e porosidade total respectivamente de 0,08 e $0,44 \mathrm{~m}^{3} \mathrm{~m}^{-3}, 2$ ) resistência à penetração de $6,1 \mathrm{MPa}$ e umidade gravimétrica de $0,13 \mathrm{~kg}$ $\mathrm{kg}^{-1}, 3$ ) densidade do solo e da partícula respectivamente de 1,28 e $2,47 \mathrm{~kg} \mathrm{dm}^{-3}$, 4) $\mathrm{pH}\left(\mathrm{CaCl}_{2}\right)$ de 4,5 e teor de matéria orgânica de $17,4 \mathrm{~g} \mathrm{dm}^{-3}$, e 5) teores de areia, silte e argila respectivamente de 504, 78 e $420 \mathrm{~g} \mathrm{~kg}^{-1}$.

Instalou-se em 10/08/2010, num talhão de 3,5ha com o Eucalyptus camaldulensis, plantado em 10/10/2008 no espaçamento de $3,0 \mathrm{~m} \times 1,8 \mathrm{~m}$, a malha para coleta de dados. Ficou constituída com seis transeções paralelas (sentido N-S), espaçadas em 15m, com 20 pontos amostrais cada, também espaçados em $15 \mathrm{~m}$. Como cada ponto representou $225 \mathrm{~m}^{2}(15 \mathrm{~m} \times 15 \mathrm{~m})$, o total deles foi de 120. Entre 15/08/2010 e 05/09/2010, coletaram-se os atributos (solo e planta) no entorno da árvore representante do ponto amostral. Os atributos do solo, nas profundidades de $0-0,20 \mathrm{~m}$ e de $0,20-0,40 \mathrm{~m}$, foram a densidade do solo (DS), $\mathrm{pH}$ em $\mathrm{CaCl}_{2}$ e o teor de matéria orgânica (MO). Com a MO e a DS calculouse o estoque de carbono do solo (EC). Os da planta foram o perímetro à altura do peito (PAP), a altura da árvore (ALT) e o diâmetro basal da copa (DBC). Com o PAPe a ALT foi calculado o volume de madeira (VOL). Os 10 atributos foram o VOL, PAP, ALT, DBC, DS1, DS2, pH1, pH2, EC1 e o EC2, onde 1 e 2 representaram respectivamente a primeira e a segunda profundidades.

Os atributos da planta foram colhidos conforme LIMA et al. (2010). O volume médio anual de madeira do fuste com a casca (VOL, em $\mathrm{m}^{3} \mathrm{ha}^{-1} \mathrm{ano}^{-1}$ ) foi dado por:

$\mathrm{VOL}=3,68444 \cdot 10^{-5}$.(PAP $\left.{ }^{2}\right)$.ALT

onde: PAPé o perímetro médio na altura do peito $(\mathrm{mm})$; ALT é a altura média da árvore $(\mathrm{m}) ; 3,68444.10^{-5}$ foi a constante dada pelo quociente [(1825.0,5.0,5)/(4p.106)], onde: a) 1825, foi para transformar o volume de madeira por árvore em volume por ha; b) 4p, para transformar raio em diâmetro; c) 106, para transformar $\mathrm{mm}^{2} \mathrm{em} \mathrm{m}^{2}$; d) 0,5, o fator de forma do Eucalyptus camaldulensis e e) 0,5 , para transformar $\mathrm{m}^{3} \mathrm{ha}^{-1} \mathrm{em} \mathrm{m}^{3} \mathrm{ha}^{-1} \mathrm{ano}^{-1}$. ODBC, em metros, foi obtido pela média dos diâmetros, um no sentido do eixo x e o outro no do y da malha. $\mathrm{O}$ pH e a $\mathrm{MO}\left(\mathrm{g} \mathrm{dm}^{-3}\right)$ foram obtidos conforme RAIJ et al. (2001), LIMAet al. (2010) e DALCHIAVON et al. (2011); a DS 
$\left(\mathrm{kg} \mathrm{dm}^{-3}\right)$, conforme a EMBRAPA (1997) e oEC $\left(\mathrm{t} \mathrm{ha}^{-1}\right)$, conforme GATTO et al. (2010). Todas as análises foram efetuadas no Laboratório de Física e Fertilidade do Solo da Faculdade de Engenharia de Ilha Solteira (SP) / UNESP.

A análise estatística foi efetuada como o SAS (SCHLOTZHAVER \& LITTELL, 1997) e a planilha Excel, seguindo os procedimentos de LIMA et al. (2010) e DALCHIAVON (2010). Foi efetuada a análise descritiva dos atributos, calculando-se a média, a mediana, os valores mínimos e máximos, o desvio padrão, o coeficiente de variação, a curtose, a assimetria, e a análise da distribuição de frequência pelo teste de Shapiro e Wilk. Foi montada a matriz de correlação entre todos os atributos pesquisados, contendo todas as combinações pareadas possíveis. Objetivou-se detectar a existência de correlações significativas entre os atributos da planta (variáveis dependentes) com os do solo (variáveis independentes). Seguidamente, efetuaram-se regressões lineares simples, do VOL em função dos atributos do solo e/ou planta, no intuito de rastrear a existência de um deles, o qual pudesse funcionar como indicador de qualidade, quando o objetivo fosse o de aumentar a produtividade de madeira.
A análise geoestatística foi feita com o Gamma Design Software $7.0\left(\mathrm{GS}^{+}, 2004\right)$, também seguindo os procedimentos de LIMA et al. (2010) e DALCHIAVON (2010). Para cada atributo foi analisada a dependência espacial, pelo cálculo do semivariograma. Efetuaram-se cokrigagens, principalmente aquelas entre o volume de madeira do eucalipto e os atributos do solo e/ou planta. O objetivo foi ratificar a existência de um atributo (solo e/ou planta) que pudesse espacialmente funcionar como indicador de qualidade, isto é, caracterizar as zonas específicas de manejo, quando o objetivo fosse o de aumentar a referida produtividade.

\section{RESULTADOS E DISCUSSÃO}

A distribuição de frequência normal, representante típica dos dados da planta, é a ideal para um estudo estatístico (análise de regressão e/ou geoestatística). Não sendo assim, busca-se a normalidade pela transformação logarítmica (MOLIN et al., 2007). Na tabela 1, o VOL apresentou distribuição de frequência lognormal, com curtose e assimetria respectivamente de $-0,425$ e 0,198 . Esse fato ficou em desacordo com a distribuição indeterminada, observada por LIMA et al. (2010) para o volume de

Tabela 1 - Análise descritiva dos componentes da produtividade do Eucalyptus camaldulensis e de alguns atributos físico-químicos de um Latossolo Vermelho Distrófico de Selvíria (MS)

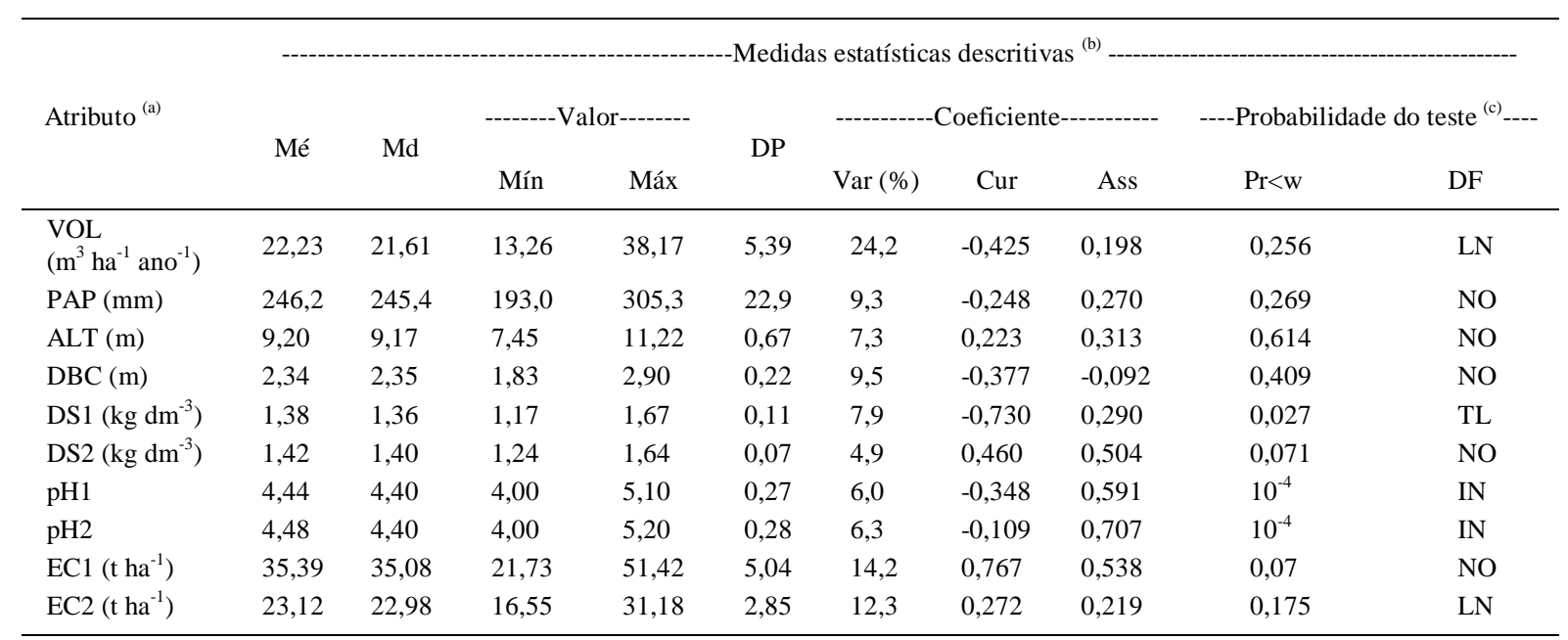

(a) VOL, PAP, ALT, e DBC são respectivamente o volume de madeira, perímetro à altura do peito, altura da árvore e o diâmetro basal da copa; DS, pH e EC são respectivamente a densidade do solo, $\mathrm{pH}$ e o estoque de carbono, nas profundidades 1 e 2 do solo; ${ }^{(\mathbf{b})} \mathrm{Mé}=$ média, Md = mediana, Mín = mínimo, Máx = máximo, $\mathrm{DP}=$ desvio padrão, Var $=$ variação, Cur $=$ curtose, Ass $=$ assimetria; ${ }^{(c)} \mathrm{DF}=$ distribuição de frequência, sendo NO, LN, TL e IN respectivamente do tipo normal, lognormal, tendendo a lognormal e indefinido.

Ciência Rural, v.42, n.10, out, 2012. 
madeira de eucalipto. Já o PAP, ALT e o DBC apresentaram-na do tipo normal, ficando, para esses mesmos atributos, de acordo com o observado por LIMA et al. (2010) e ROSA FILHO et al. (2011). Ficaram também de acordo com as distribuições normais observadas por LIMA et al. (2007), ANDREOTTI et al. (2010), MONTANARI et al. (2010) eBASSO et al. (2011), quando pesquisaram produtividade vegetal, segundo preceitos geoestatísticos, para culturas anuais e forrageiras.

Houve baixa variabilidade dos dados para o PAP, ALT, DBC, DS1, DS2, pH1 e pH2, analisada pela magnitude do coeficiente de variação, estabelecida por PIMENTEL-GOMES \& GARCIA (2002). Já o EC e o VOL a revelaram respectivamente como média e alta. $\mathrm{O}$ volume mediano de madeira $\left(21,6 \mathrm{~m}^{3} \mathrm{ha}^{-1} \mathrm{ano}^{-1}\right)$, quando comparado aos $45 \mathrm{~m}^{3} \mathrm{ha}^{-1}$ ano $^{-1}$, do gênero Eucalyptus spp. para as condições brasileiras (LIMA et al., 2010), foi baixo devido à idade juvenil do talhão trabalhado (2 anos). Os valores da densidade do solo em profundidade $\left(1,38-1,42 \mathrm{~kg} \mathrm{dm}^{-3}\right)$ indicaram que o solo se encontrava compactado (Tabela 1), uma vez que, conforme observado por MONTANARI et al. (2010), a densidade dos solos argilosos em condições virgens normalmente varia entre $0,98-1,13 \mathrm{~kg} \mathrm{dm}^{-3}$.

Houve correlação significativa para os pares de atributos: 1) VOL x PAP (r=0,95**), 2) VOL x ALT $\left.\left(\mathrm{r}=0,85^{* *}\right), 3\right)$ VOL $x$ DBC $\left.\left(\mathrm{r}=0,68^{* *}\right), 4\right)$ VOL $x$ DS2 ( $\mathrm{r}=-$ $0,19 *)$, 5) PAP x ALT $(\mathrm{r}=0,77 * *), 6)$ PAP x DBC $\left.\left(\mathrm{r}=0,70^{* *}\right), 7\right)$ PAP x DS2 $\left.\left(\mathrm{r}=-0,20^{*}\right), 8\right)$ ALT $x$ DBC $\left.\left(\mathrm{r}=0,64^{* *}\right) \mathrm{e} 9\right)$ ALT $x$ DS2 $\left(\mathrm{r}=-0,19^{*}\right)$. Como mencionado, o PAP e a ALT, por serem fatores do termo-produto VOL, são variáveis dependentes que estabelecem clara relação de interdependência com ele. Por consequência, os coeficientes de correlação dos dois primeiros pares (VOLx PAPe VOLx ALT) foram elevados e positivos, denotando apreciável relação direta entre os atributos envolvidos, corroborando o que havia sido observado por LIMA et al. (2010). Já o DS2 foi o único atributo do solo significativo com o VOL, com um coeficiente de correlação baixo e negativo, indicando relação inversa entre eles. Sua baixa magnitude $\left(r=-0,19^{*}\right)$, obtida segundo preceitos da análise geoestatística e que havia sido previamente comentada por MONTANARI et al. (2010), foi devido basicamente a dois fatos: a) exemplo clássico de variável dependente (VOL) contra independente (DS2) e b) elevado número de observações $(n=120)$. Tal fato é uma realidade em relação a tais estudos que normalmente trabalham com muitos dados para se compor as regressões, assim como para a modelagem da dependência espacial (LIMA et al., 2007; MOLIN et al., 2007; ANDREOTTI et al., 2010; MONTANARI et al., 2010; BASSO et al., 2011). Assim, as equações ajustadas foram:

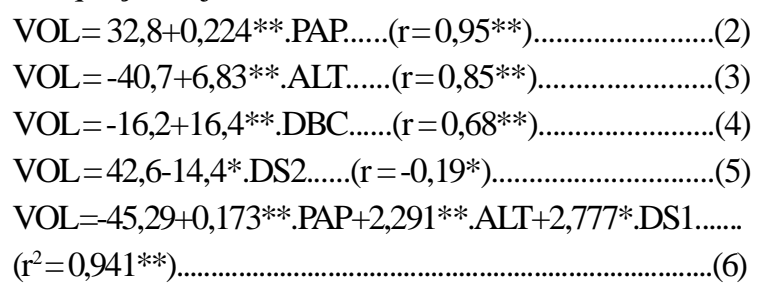

As equações (2) e (3) revelaram influência linear direta do PAPe da ALT sobre o VOL. A melhor, eq. (2), diferiu do modelo ajustado por LIMA et al. (2010), que a revelaram na forma potencial. As eq. (4) e (5) apresentaram respectivamente variação direta e inversa também na forma linear com o VOL. Suas variáveis independentes (DBC e DS2), por não possuírem qualquer relação de interdependência com a variável dependente (VOL), ainda que tenham revelado uma correlação entre média e baixa, poderão funcionar como indicadores de qualidade, quando o objetivo for aumentar o volume de madeira de eucalipto (VOL). Em relação à eq. (5), sua variação inversa ficou de acordo àquelas obtidas por LIMA et al. (2007) e BASSO et al. (2011), quando pesquisaram correlações da produtividade vegetal com a densidade do solo. Também evidenciou haver necessidade de se descompactar o solo pesquisado para proporcionar aumento no VOL. A regressão linear múltipla pelo step wise apresentou pequeno aumento do VOL em função do PAP, ALT e DS1, dada pela eq. (6).

A análise geoestatística (Tabela 2, Figura 1) mostrou, para os atributos que não resultaram efeito pepita puro, que os atributos da planta apresentaram coeficientes de correlação espacial $\left(\mathrm{r}^{2}\right)$ muito altos (0,965-0,974), dependências espaciais (ADE) entre média a muito alta $(59,5-91,0 \%)$ e coeficientes angulares (b) da validação cruzada (Tabela 3) próximos da unidade $(0,883-0,986)$. Esses dados foram muito semelhante àqueles obtidos por LIMA et al. (2010), quando foi pesquisada a mesma espécie de eucalipto. Os atributos do solo apresentaram coeficientes de correlação espacial entre médio $(0,733)$ e muito alto $(0,994)$, dependências espaciais também entre média $(50,0 \%)$ a 
Tabela 2 - Parâmetros dos semivariogramas dos componentes da produtividade do Eucalyptus camaldulensis e de alguns atributos físicoquímicos de um Latossolo Vermelho Distrófico de Selvíria (MS)

\begin{tabular}{|c|c|c|c|c|c|c|c|c|}
\hline \multirow{2}{*}{ Atributo $^{(a)}$} & \multirow{2}{*}{ Modelo $^{(b)}$} & \multirow{2}{*}{$\mathrm{C}_{\mathrm{o}}$} & \multirow{2}{*}{$\mathrm{C}_{\mathrm{o}}+\mathrm{C}$} & \multirow{2}{*}{$\mathrm{A}_{\mathrm{o}}(\mathrm{m})$} & \multirow{2}{*}{$r^{2}$} & \multirow{2}{*}{$\mathrm{SQR}^{(\mathrm{c})}$} & \multicolumn{2}{|c|}{$\mathrm{ADE}^{(\mathrm{d})}$} \\
\hline & & & & & & & $\%$ & Classe \\
\hline \multicolumn{9}{|c|}{ - } \\
\hline VOL & gau (352) & 6,16 & $2,67.10$ & 73,8 & 0,974 & 8,25 & 76,9 & $\mathrm{AL}$ \\
\hline PAP & esf (392) & $5,00.10$ & $5,56.10^{2}$ & 71,6 & 0,974 & $2,65.10^{3}$ & 91,0 & MA \\
\hline ALT & gau (353) & $1,63.10^{-1}$ & $4,02.10^{-1}$ & 75,7 & 0,965 & $1,56.10^{-3}$ & 59,5 & ME \\
\hline $\mathrm{DBC}$ & $\exp (202)$ & $1,74.10^{-2}$ & $5,24.10^{-2}$ & 176,1 & 0,983 & $1,18.10^{-5}$ & 66,8 & $\mathrm{AL}$ \\
\hline DS2 & $\exp (190)$ & $1,14.10^{-3}$ & $3,74.10^{-3}$ & 60,0 & 0,733 & $6,27 \cdot 10^{-7}$ & 69,6 & $\mathrm{AL}$ \\
\hline pH1 & $\exp (371)$ & $1,84.10^{-2}$ & $5,27 \cdot 10^{-2}$ & 124,2 & 0,994 & $6,46.10^{-6}$ & 74,1 & $\mathrm{AL}$ \\
\hline $\mathrm{pH} 2$ & $\exp (214)$ & $1,02 \cdot 10^{-2}$ & $8,31 \cdot 10^{-2}$ & 78,9 & 0,962 & $7,97 \cdot 10^{-5}$ & 87,7 & MA \\
\hline EC1 & $\exp (197)$ & 7,43 & $2,20.10$ & 73,8 & 0,959 & 2,53 & 66,2 & $\mathrm{AL}$ \\
\hline $\mathrm{EC} 2$ & $\operatorname{esf}(200)$ & 3,81 & 7,62 & 78,3 & 0,874 & 1,06 & 50,0 & ME \\
\hline & & & ------Sem & grama cr & - & 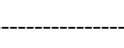 & & --- \\
\hline $\mathrm{VOL}=\mathrm{f}(\mathrm{ALT})$ & gau (328) & 5,87 & $2,38.10$ & 74,5 & 0,980 & 4,75 & 75,3 & $\mathrm{AL}$ \\
\hline $\mathrm{VOL}=\mathrm{f}(\mathrm{DBC})$ & $\exp (337)$ & $1,00.10^{-3}$ & $9,24.10^{-1}$ & 197,4 & 0,969 & $1,50.10^{-2}$ & 99,9 & MA \\
\hline $\mathrm{VOL}=\mathrm{f}(\mathrm{DS} 2)$ & gau (164) & $-1,00.10^{-4}$ & $-5,60.10^{-2}$ & 66,9 & 0,684 & $1,61.10^{-3}$ & 99,8 & MA \\
\hline $\mathrm{VOL}=\mathrm{f}(\mathrm{EC} 2)$ & gau (330) & $-1,00.10^{-3}$ & $-16,73 \cdot 10^{-1}$ & 95,6 & 0,694 & 1,01 & 99,9 & MA \\
\hline
\end{tabular}

(a) idem Tabela 1; ${ }^{\text {(b) }}$ esf = esférico, exp = exponencial e gau = gaussiano; algarismos entre parênteses significando número de pares no primeiro lag; ${ }^{(\mathbf{c})} \mathrm{SQR}=$ soma dos quadrados dos resíduos; ${ }^{(\mathbf{d})} \mathrm{ADE}=$ avaliador da dependência espacial, $\mathrm{AL}=$ alta, $\mathrm{MA}=$ muito alta e ME = média.

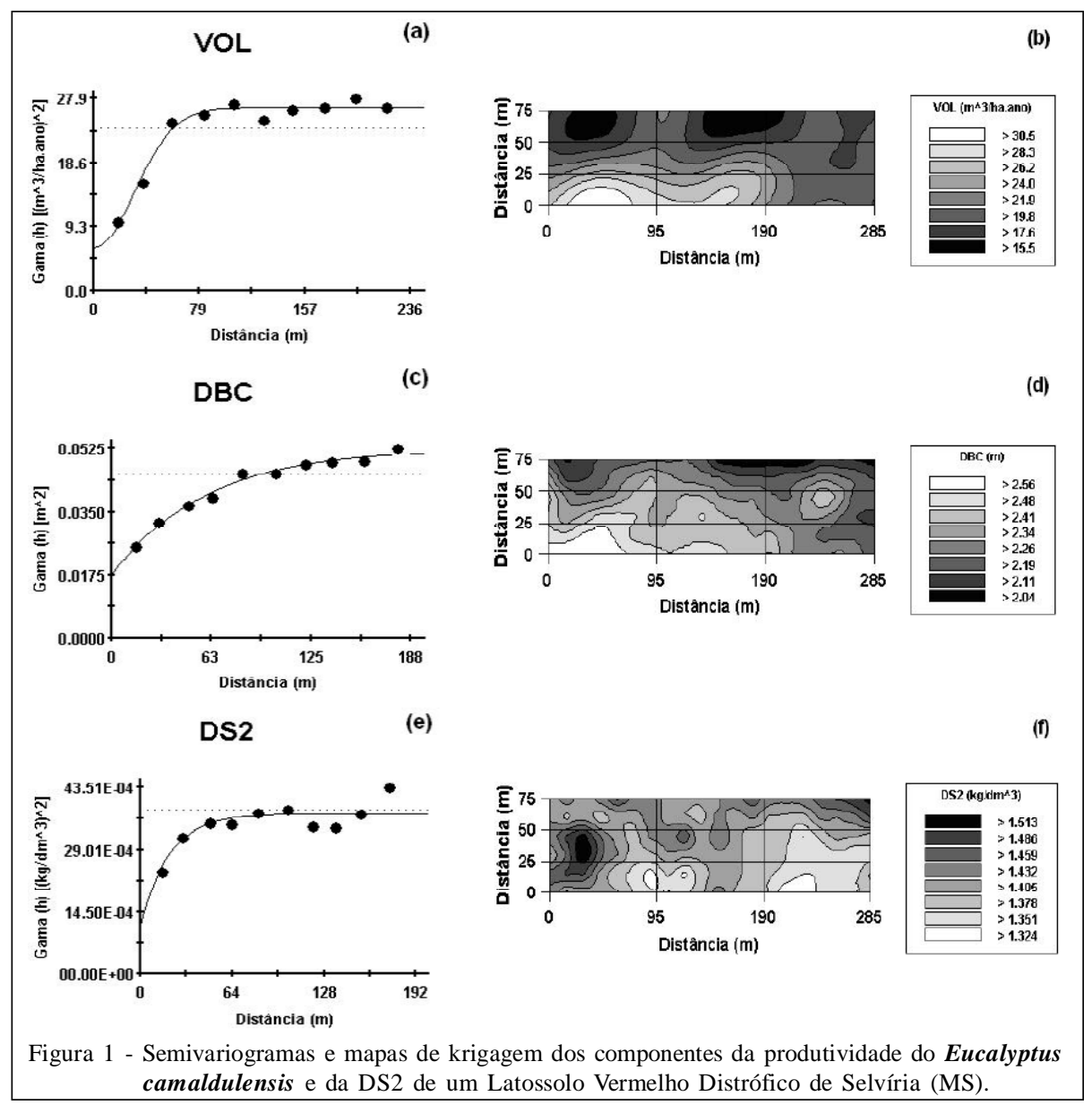

Ciência Rural, v.42, n.10, out, 2012. 
Tabela 3 - Parâmetros das validações cruzadas dos componentes da produtividade do Eucalyptus camaldulensis e de alguns atributos físico-químicos de um Latossolo Vermelho Distrófico de Selvíria (MS)

\begin{tabular}{|c|c|c|c|}
\hline \multirow{2}{*}{ Atributo $^{(a)}$} & \multicolumn{3}{|c|}{------Parâmetros da validação cruzada ${ }^{(b)}$} \\
\hline & $\mathrm{a}$ & $\mathrm{b}$ & $\mathrm{r}$ \\
\hline & \multicolumn{3}{|c|}{ 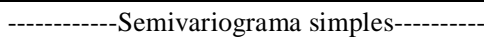 } \\
\hline VOL & 1,27 & 0,941 & 0,765 \\
\hline PAP & $2,88.10$ & 0,883 & 0,696 \\
\hline ALT & $1,30 \cdot 10^{-1}$ & 0,986 & 0,677 \\
\hline DBC & $-7,00.10^{-2}$ & 1,028 & 0,609 \\
\hline DS2 & $-2,00 \cdot 10^{-3}$ & 1,002 & 0,538 \\
\hline pH1 & 0 & 1,000 & 0,637 \\
\hline $\mathrm{pH} 2$ & $-2,00 \cdot 10^{-2}$ & 1,005 & 0,678 \\
\hline $\mathrm{EC} 1$ & $4,25 \cdot 10^{-2}$ & 1,001 & 0,468 \\
\hline \multirow[t]{2}{*}{$\mathrm{EC} 2$} & 2,31 & 0,904 & 0,439 \\
\hline & \multicolumn{3}{|c|}{----------Semivariograma cruzado-------- } \\
\hline $\mathrm{VOL}=\mathrm{f}(\mathrm{ALT})$ & 3,47 & 0,840 & 0,718 \\
\hline $\mathrm{VOL}=\mathrm{f}(\mathrm{DBC})$ & 2,88 & 0,870 & 0,748 \\
\hline $\mathrm{VOL}=\mathrm{f}(\mathrm{DS} 2)$ & 3,09 & 0,860 & 0,740 \\
\hline $\mathrm{VOL}=\mathrm{f}(\mathrm{EC} 2)$ & 4,17 & 0,806 & 0,725 \\
\hline
\end{tabular}

(a) idem Tabela 1; ${ }^{\text {(b) }}$ coeficientes da validação cruzada.

muito alta $(87,7 \%)$ e coeficientes angulares muito próximos da unidade $(0,904-1,005)$.

Os semivariogramas cruzados (Tabela 2, Figura 2) apresentaram elevados coeficientes de determinação espacial $\left(\mathrm{r}^{2}\right)$ para as variáveis secundárias
ALT e DBC $(0,969-0,980)$, assim como médios para as do solo, dados pela DS2 e EC2 $(0,684-0,694)$, ficando de acordo com LIMA et al. (2007) ANDREOTTI et al. (2010), MONTANARI et al. (2010) BASSO et al. (2011). Assim, do ponto de vista geoestatístico (Figura 1), ocorreram apreciáveis correlações espaciais do VOL com o DBC (direta) e com a DS2 (inversa).

Nas figuras 1 e 2 , as cokrigagens $\mathrm{VOL}=\mathrm{f}(\mathrm{DBC})$ e $\mathrm{VOL}=\mathrm{f}(\mathrm{DS} 2)$ apresentaram, nos sítios de menores DBC $(2,04-2,26 \mathrm{~m})$, coincidentes com os maiores DS2 $\left(1,432-1,513 \mathrm{~kg} \mathrm{dm}^{-3}\right)$, os menores VOL $\left(15,5-21,9 \mathrm{~m}^{3} \mathrm{ha}^{-1} \mathrm{ano}^{-1}\right)$. Já nos de maiores DBC $(2,34-$ $2,56 \mathrm{~m})$, coincidentes com os de menores DS2 (1,324$\left.1,405 \mathrm{~kg} \mathrm{dm}^{-3}\right)$, os maiores VOL $\left(24,0-30,5 \mathrm{~m}^{3} \mathrm{ha}^{-1}\right.$ ano $\left.^{-1}\right)$. Portanto, o DBC e a DS2, por terem revelado estreita relação espacial com o VOL, tiveram bom desempenho no delineamento de duas zonas especificas de manejo do eucalipto. Assim, eles podem ser utilizados como indicadores do VOL, quando a intenção for a de aumentar o volume de madeira do eucalipto. Esse fato foi semelhante aquele de LIMA et al. (2010), os quais relacionaram correlações espaciais inversas do volume de madeira do eucalipto com a resistência mecânica à penetração e o pH do solo.

\section{CONCLUSÃO}

O diâmetro basal copa e a densidade do solo, por resultarem estreitas correlações com o volume de madeira, foram indicadores de zonas específicas de manejo intimamente associadas à produção de madeira do eucalipto.

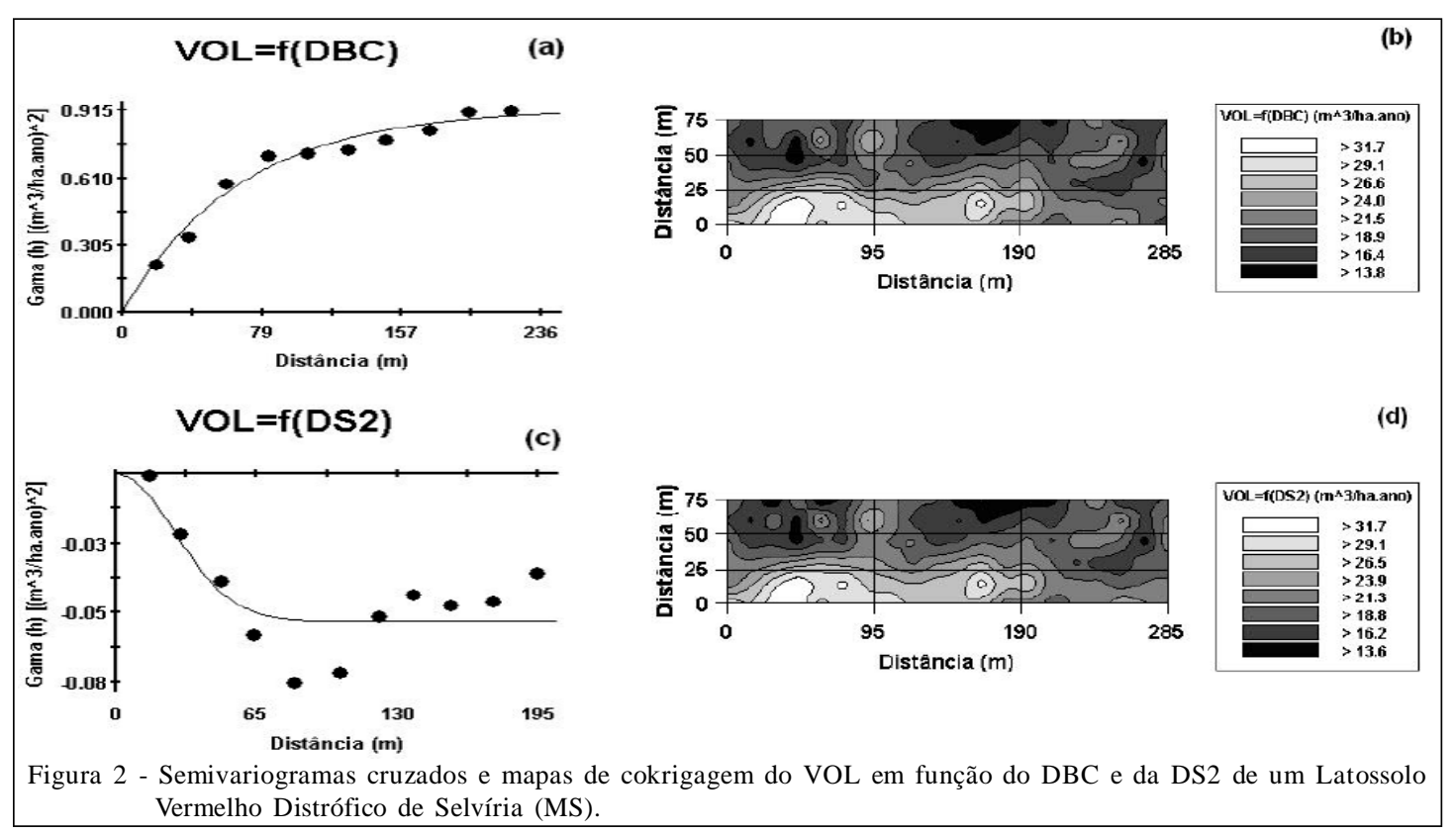

Ciência Rural, v.42, n.10, out, 2012. 


\section{REFERÊNCIAS}

ANDREOTTI, M. et al. Produtividade da soja correlacionada com a porosidade e a densidade de um Latossolo Vermelho do Cerrado Brasileiro. Ciência Rural, Santa Maria, v.40, n.3, p.520-526, 2010. Disponível em: <http://www.scielo.br/ scielo.php? script $=$ sci_abstract \& pid=S0103. $84782010000300004 \& \operatorname{lng}=\mathrm{pt} \& \mathrm{nrm}=\mathrm{iso} \& \mathrm{t} \operatorname{lng}=\mathrm{pt}>$. Acesso em: 20 maio, 2011. doi: 10.1590/S0103-84782010005000032.

BASSO, F.C. et al. Relações entre produtividade de sorgo forrageiro e atributos físicos e teor de matéria orgânica de um latossolo do Cerrado. Pesquisa Agropecuária Tropical, Goiânia, v.41, n.1, p.135-144, 2011. Disponível em: <http:// www.revistas.ufg.br/index.php/pat/article/view/7099>. Acesso em: 18 jun. 2011. doi: 10.5216/pat.v41i1.7099.

DALCHIAVON, F.C. Aspectos da produtividade do arroz de terras altas irrigado com atributos químicos do solo sob plantio direto. 2010. 112f. Dissertação (Mestrado em Agronomia) - Curso de Pós-graduação em Agronomia, Universidade Estadual Paulista, SP.

EMPRESA BRASILEIRA DE PESQUISA AGROPECUÁRIA EMBRAPA. Centro Nacional de Pesquisa de Solos. Manual de métodos de análise do solo. 2.ed. Rio de Janeiro, 1997. 212 p.

EMPRESA BRASILEIRA DE PESQUISA AGROPECUÁRIA EMBRAPA. Sistema brasileiro de classificação de solos. 2.ed. Rio de Janeiro, 2006. 306p.

GATTO, A. et al. Estoques de carbono no solo e na biomassa em plantações de eucalipto. Revista Brasileira de Ciência do Solo, Viçosa, v.34, n.4, p.1069-1079, 2010. Disponível em: <http://www.scielo.br/scielo.php?script=sci_abstract\&pid=S0100$06832010000400007 \& \operatorname{lng}=\mathrm{pt} \& \mathrm{nrm}=\mathrm{iso} \& \mathrm{t} \operatorname{lng}=\mathrm{pt}>$. Acesso em: 10 abr. 2011. doi: 10.1590/S0100-06832010000400007.

GS+: GAMMA DESIGN SOFTWARE. Geostatistics for the environmental sciences. 7.ed. Plainwell: Gamma Design Software. 2004. 159p.

LIMA, C.G.R. et al. Correlação linear e espacial entre a produtividade de forragem, a porosidade total e a densidade do solo de Pereira Barreto. Revista Brasileira de Ciência do Solo, Viçosa, v.31, n.6, p.1233-1244, 2007. Disponível em: <http://www.scielo.br/scielo.php?script=sci_abstract\&pid=S0100-
$06832007000600002 \& \operatorname{lng}=\mathrm{en} \& \mathrm{nrm}=\mathrm{iso} \& \mathrm{t} \operatorname{lng}=\mathrm{pt}>$. Acesso em: 08 abr. 2011. doi: 10.1590/S0100-06832007000600002.

LIMA, C.G.R. et al. Atributos físico-químicos de um Latossolo do cerrado brasileiro e sua relação com características dendrométricas do eucalipto. Revista Brasileira de Ciência do Solo, Viçosa, v.34, n.1, p.163-173, 2010. Disponível em: $<$ http://www.scielo.br/scielo.php?script=sci_abstract\&pid=S0100$06832010000100017 \& \operatorname{lng}=$ en $\& n r m=i s o \& t \operatorname{lng}=p t>$. Acesso em: 18 jun. 2011. doi: 10.1590/S0100-06832010000100017.

MOLIN, J.P. et al. Variação espacial na produtividade de milho safrinha devido aos macronutrientes e à população de plantas. Revista Brasileira de Milho e Sorgo, Sete Lagoas, v.6, n.3, p.309-324, 2007. Disponível em: <http://rbms.cnpms.embrapa.br/ index.php/ojs/article/view/235/240>. Acesso em: 08 maio, 2011.

MONTANARI, R. et al. Aspectos da produtividade do feijão correlacionados com atributos físicos do solo sob elevado nível tecnológico de manejo. Revista Brasileira de Ciência do Solo, Viçosa, v.34, n.6, p.1811-1822, 2010. Disponível em: <http://www.scielo.br/scielo.php?script=sci_abstract\&pid=S0100$06832010000600005 \& \operatorname{lng}=\mathrm{en} \& \mathrm{nrm}=\mathrm{iso} \& \mathrm{t} \operatorname{lng}=\mathrm{pt}>$. Acesso em: 18 maio, 2011. doi: 10.1590/S0100-06832010000600005.

PIMENTEL-GOMES, F.; GARCIA, C.H. Estatística aplicada a experimentos agronômicos e florestais: exposição com exemplos e orientações para uso de aplicativos. 11.ed. Piracicaba: FEALQ, 2002. 309p.

RAIJ, B. van et al. Análise química para avaliação da fertilidade de solos tropicais. Campinas: Instituto Agronômico, 2001. 285p.

ROSA FILHO, G. et al. Variabilidade espacial de propriedades dendrométricas do eucalipto e de atributos físicos de um Latossolo Vermelho. Bragantia, Campinas, v.70, n.2, p.439446, 2011. Disponível em: <http://www.scielo.br/ s c i e lo.ph p s c ri p t $=$ s c i $\_$pd f \& pi d = S 0006 $87052011000200027 \& \operatorname{lng}=$ pt\&nrm $=$ iso\&tlng=pt $>$. Acesso em: 24 out. 2011 .

SCHLOTZHAVER, S.D.; LITTELL, R.C. SAS system for elementary statistical analysis. 2.ed. Cary: SAS Institute, 1997. $441 \mathrm{p}$.

TSCHIEDEL, M.; FERREIRA, M.F. Introdução à agricultura de precisão: conceitos e vantagens. Ciência Rural, Santa Maria, v.32, n.1, p.159-163, 2002. Disponível em: <http:// www.scielo.br/scielo.php?script $=$ sci_abstract \&pid $=$ S0103$84782002000100027 \& \operatorname{lng}=p t \& n r m=i s o \& t \operatorname{lng}=p t>$. Acesso em: 20 maio, 2011. doi: 10.1590/S0103-84782002000100027. 